\title{
End-to-end efficiency quantification of an autonomous underwater vehicle propulsion system
}

\author{
Artur K. Lidtke a,b,*, Nicholas P. Linton ${ }^{\mathrm{c}}$, Hannah L. Wright ${ }^{\mathrm{c}}$, Stephen R. Turnock ${ }^{\mathrm{a}}$, Jon Downes ${ }^{\mathrm{a}}$ \\ ${ }^{a}$ Fluid Structure Interactions Group, University of Southampton, SO16 7QF, United Kingdom \\ ${ }^{\mathrm{b}}$ Maritime Research Institute Netherlands (MARIN), 2 Haagsteeg, 6708 PM, Wageningen, The Netherlands \\ ${ }^{\mathrm{c}}$ Marine Autonomous and Robotic Systems Group, National Oceanography Centre, European Way, Southampton SO14 3ZH, United Kingdom
}

\section{A R T I C L E I N F O}

\section{Keywords:}

AUV

Hydrodynamics

Propulsion

\begin{abstract}
A B S T R A C T
Increasing demand for versatile and long-endurance autonomous underwater vehicles puts significant design pressure on all aspects of AUV design and operation, including that of the propulsive system. The present study discusses testing of a thruster unit and several propellers developed to propel a hybrid glider/flight-style underwater vehicle. Due to the AUV being required to operate at largely different speeds and thrust levels between the two configurations, the propulsive subsystem needs to be capable of remaining efficient and effective across a wide range of operating conditions. Thus, the current results focus on quantifying all of the factors affecting the drive train, ranging from open-water performance of the propeller up to electro-mechanical efficiency of the magnetic coupling and geared electric motor. It is shown that, depending on the required operating point, total efficiency of the vehicle is primarily affected by non-linear low Reynolds number effects, sudden drop of gearbox efficiency at low revolutions and applied torques, as well as blade deformation, aside of the baseline propeller efficiency.
\end{abstract}

\section{Introduction}

Underwater gliders are autonomous underwater vehicles (AUV) that rely on using a buoyancy engine in order to ascend or descend through the water column. By adjusting their pitch they can utilise this vertical motion in order to develop forward thrust from their hydrofoils. This propulsion method allows them to undertake long-endurance missions, often many weeks long (Graver, 2005; Rudnick et al., 2004; Eriksen, 2003). However, their mode of locomotion renders their speed and rate of ascent susceptible to currents, water density variations, and hull compressibility. This adversely affects their ability to perform measurements at a particular location as well as increases uncertainty errors associated with dead reckoning and localisation of the vehicle between GPS fixes obtained at the surface. Furthermore, being reliant on the buoyancy engine alone limits the types of missions gliders are able to perform. For this reason, several vehicles in use today employ small thruster units intended to provide an additional means of propulsion. However, they are used mostly for overcoming currents and maintaining steady rates of ascent or descent rather than to provide the ability to operate the vehicle as a conventional AUV (Claus et al., 2010; Chen et al., 2016; Isa et al., 2014). Thrusters used on ROVs or conventional AUVs typically need to be operated over a wide range of conditions and inflow angles, making even omni-directional performance an important aspect of the design (Kim and Chung, 2006; Allotta et al., 2015; Pugi et al., 2018).

Present work discusses the design, manufacture and testing of a thruster unit designed to provide secondary means of propelling the BRIDGES (http://www.bridges-h2020.eu/) Deep Explorer underwater glider as well as allowing it to operate as a conventional flightstyle AUV. Such a configuration of the vehicle is expected to enable a much wider operational envelope than either contemporary underwater gliders or propeller-driven AUVs. Design of the thruster had to balance several often conflicting requirements, such as minimisation of drag while gliding, efficient operation at very low thrust levels when assisting the glider, being able to deliver sufficient power to propel the vehicle when it is to operate as a conventional AUV, as well as flexibility in matching power output to variable configurations and drag of the glider.

Compared to ships, the designed propellers are characterised by relatively low loading and small blade area ratio (BAR), making them more akin to airborne drone propellers. This is caused by relatively high rpm of the electric motors compared to diesel engines, which poses practical limits on maximum blade area ratio due to maximum power output of the prime mover. The necessity to operate in a wake

\footnotetext{
* Corresponding author at: Maritime Research Institute Netherlands (MARIN), 2 Haagsteeg, 6708 PM, Wageningen, The Netherlands. E-mail addresses: A.Lidtke@soton.ac.uk, A.Lidtke@marin.nl (A.K. Lidtke).
} 


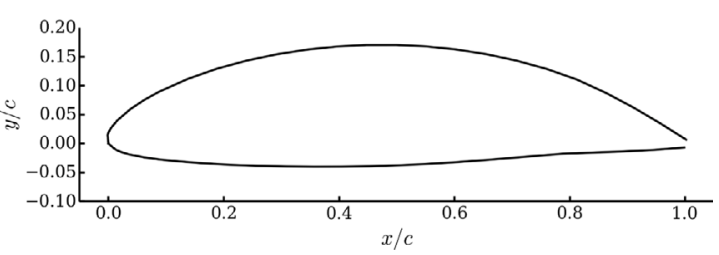

Fig. 1. NACA 66 mod section offsets used for the propeller.

field, at much lower advance speeds, and in a different medium cause the operational envelopes of the present and airborne propellers to differ significantly. Furthermore, the required rpm and torque levels necessitate the use of a gearbox in the AUV drive train, introducing an additional efficiency term that needs to be understood and optimised for an efficient design.

Developing a detailed understanding of the aforementioned effects and using that knowledge for the design of an effective propulsor is the key focus of the present study. This is done through quantification of the motor-gearbox efficiency, towing tank experiments involving of a series of 3D-printed propellers designed to deliver various levels of thrust, and basic measurements of blade deformation under load.

\section{Propulsion unit design}

\subsection{Design requirements}

The designed propeller was required to deliver approximately $20 \mathrm{~N}$ of thrust, which had been estimated based on wind tunnel measurements (Lidtke et al., 2018) and numerical predictions (Lidtke et al., 2017). The nominal wake fraction was estimated using CFD and yielded average nominal inflow speeds of 0.13 and $0.44 \mathrm{~m} \mathrm{~s}^{-1}$ for vehicle speeds of 0.3 and $1.0 \mathrm{~m} \mathrm{~s}^{-1}$, respectively. These correspond to cruise and sprint conditions, with the former also being representative of glide-assist operation mode of the propeller.

Large difference between the cruise and sprint conditions implied an equally wide range of thrust requirements and operating points. Thus, while the key design point was taken to be the sprint condition in order to ensure the vehicle will be able to reach its desired speed, consideration also had to be taken of the thrust developed at lower velocities. The design was also further complicated by a maximum electrical power requirement of $10 \mathrm{~W}_{e}$ at the cruise condition. Evaluating the degree to which this specification has been met necessitated careful accounting for the different flow conditions expected at that vehicle speed. Furthermore, the interplay of hydrodynamic and electromechanical efficiencies had to be taken into consideration to guarantee the endurance target of the vehicle would be met.

\subsection{Propeller design}

NACA 66 mod profile was selected for the propeller blades, offsets of which are shown in Fig. 1. This blade profile is widely used on marine propellers due to its favourable cavitation avoidance and structural characteristics (Carlton, 2012). While the former is not of concern here due to the vehicle typically operating at high static pressures, the relatively thick trailing edge and large cross-sectional area of the foil were deemed favourable due to the intention to manufacture the propeller blades using 3D printing.

Preliminary design of the propeller was carried out using blade element momentum theory (BEMT) (Molland et al., 2011). Due to its inexpensive nature, a wide range of solutions could be evaluated while considering the effects of diameter, pitch, chord, camber, and number of blades. Hydrodynamic performance of each propeller was also assessed in conjunction with structural characteristics to ensure the designs could be feasibly manufactured. Following several design

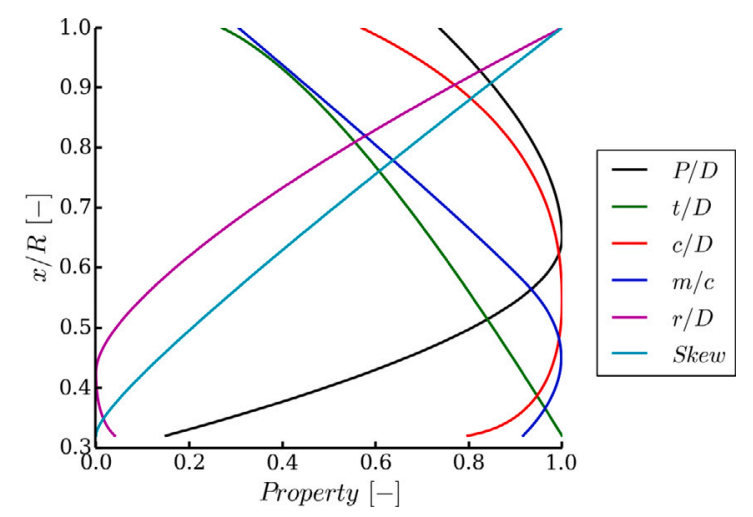

Fig. 2. Radial distributions of geometric characteristics of the propeller blades - pitch, thickness, chord, camber, rake and skew. Each curve is divided by the maximum value.

Table 1

Nominal values of the propeller characteristics used to dimensionalise distributions shown in Fig. 2.

\begin{tabular}{lc}
\hline Parameter & Value \\
\hline Diameter, D [mm] & 250.0 \\
Chord-diameter ratio, c/D & 0.1250 \\
Thickness-diameter ratio, t/D & 0.0235 \\
Maximum camber to chord ratio, m/c & 0.0325 \\
Rake/D & 0.0450 \\
Skew angle [deg] & 15.0 \\
Hub ratio & 0.32 \\
\hline
\end{tabular}

iterations using the numerical tools, a target design was proposed with key parameters as depicted in Fig. 2 and Table 1. A design pitch value of 0.35 was also selected.

However, it was recognised that the used numerical tools might be prone to substantial prediction errors stemming from the difficulty of accurately accounting for the effects of laminar-turbulent transition as a function of blade Reynolds number, surface roughness, and onset turbulence levels. It was also sought to measure performance of a range of propellers in order to provide a suite of choices for the vehicle operating with different payload configurations. Therefore, a range of designs with the same other design parameters and constant pitch distributions but varying nominal pitch values were designed, with P/D of $0.25,0.35,0.45,0.55$ and 0.60 . This was done to evaluate the effect of changing angle of attack of the blade, which was expected to have the most significant effect on the performance of the propeller in the expected operating regimes. A rendering of one of the blade geometries is depicted in Fig. 3

A characteristic feature of the current design is its ability to unfold using rotational inertia and hydrodynamic moment developed by the tip of the blade. This is facilitated by the root of the blade being mounted on a pin that allows free rotation and stopping elements on the hub which restrict the range of motion. This should allow the propeller to exert a minimum drag augment on the vehicle when it is gliding but give it the ability to deploy the propeller with the rotational action of the shaft alone. In order to allow the blade to develop a sufficient moment around the root, relatively large pitch values and, consequently, angles of attack had to be adopted near the tip. This is expected to reduce the efficiency of the blade but is a necessary sacrifice to meet the functional requirements without employing complex and potentially less reliable mechanical unfolding systems. The unfolding ability was further enhanced by placing the pivot axis at an angle to the horizontal plane, thereby increasing the pitch of the entire blade when folded.

Selective laser sintering (SLS) 3D-printing using Nylon-12 has been selected for making the blades. This process has been successfully used on underwater vehicles before and offers a cost-effective and flexible solution for manufacturing of complex shapes. To improve surface 


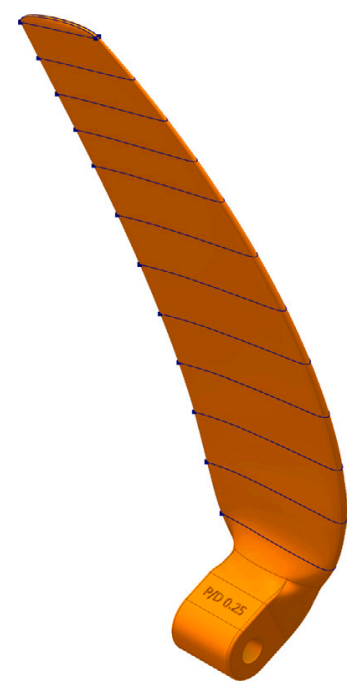

Fig. 3. Rendering of one of the tested propeller blades, also showing sections used to generate the shape as well as the root arrangement designed to provide folding capability. Hole for the mounting pin is placed at an angle to the shaft axis in order to facilitate easier unfolding.

finish of the manufactured blades and provide additional protection against moisture absorption, the finished prints may also polished and dyed with little added cost or complexity. The selected material is, however, characterised by lower strength and stiffness than, for instance, composite materials, which are often used on aircraft propellers of similar size (Brandt and Selig, 2011). Thus, it has been found necessary to keep the minimum trailing edge thickness of at least $0.6 \mathrm{~mm}$ at the tip of the blade and $1.0 \mathrm{~mm}$ at the root in order to ensure satisfactory print quality and level of detail of the finished print. Furthermore, the minimum blade thickness of $1.5 \mathrm{~mm}$ has been adopted to meet structural requirements.

These have been assessed using finite element analysis by prescribing a uniform pressure of $200 \mathrm{~kg} / \mathrm{m}^{2}$ in the thrust direction, which is an equivalent of load of approximately $100 \mathrm{~N}$, or five times the design thrust of the propeller. The design was considered to meet the structural requirements if the maximum stress did not exceed $35 \mathrm{MPa}$, which is the tensile strength of the material used. A second criterion was also considered whereby an equivalent of design thrust was applied to the blade and it was ensured that the maximum stress does not exceed $10 \mathrm{MPa}$ to avoid creep failure.

Structural analysis results were also used to determine maximum deflection of the blade, which for the extreme load case was estimated to reach up to $12 \mathrm{~mm}$ for some of the blade designs. To compensate for this and protect against the blades impacting the upstream part of the vehicle, a linear rake distribution was applied to the propeller. This ensured that as long as the load on the blades remains within structurally feasible limits, sufficient clearance will exist between the face of the propeller and the appendages.

It has been reported that accurate predictions of flow past flexible wings and propellers can be made by carrying out fluid-structure interaction simulations which solve for both structural response and fluid loading simultaneously (Giovannetti et al., 2016, 2018; Maljaars et al., 2018). However, such simulations were beyond the scope of the current investigation and would also require reliable validation data for rotating geometries, which have proven difficult to obtain. Thus it was aimed to rely on the experimental results to quantify the effect of the blade deformation on performance.

\subsection{Thruster design}

The thruster unit has been designed to operate safely at depths up to $6000 \mathrm{~m}$. To simplify installation and maintenance, an early decision

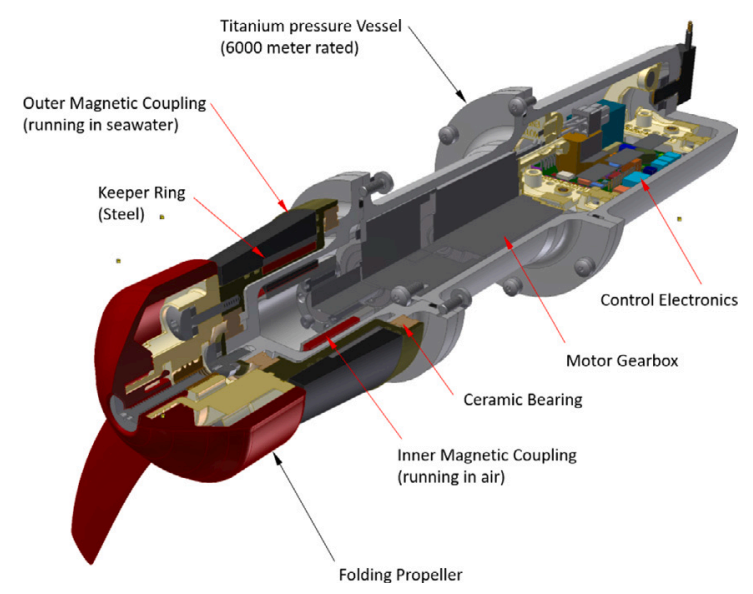

Fig. 4. Overall layout of the thruster unit.

was taken to house the thruster in an air filled pressure vessel, thus eliminating the need for additional oil compensator units. This pressure vessel was manufactured from titanium (grade 5) and pressure-tested at the National Oceanography Centre. The housing comprises of three segments, housing the electronics, the motorgearbox system, and the internal part of the radial magnetic coupling, as shown in Fig. 4. Oring seals are used between each cement of the housing, but in practice only one of them needs to be opened for servicing due to all of the electronics being housed on a single, easily detachable internal frame.

The use of a magnetic coupling transferring motor torque through the titanium housing eliminates the need for a shaft seal, greatly increasing reliability. The outer magnetic coupling runs in sea water, with magnet units bonded into a titanium outer mandrel before being sealed within a cast polyurethane encapsulant. Also contained within the polyurethane is a steel keeper ring, which, by redirecting magnetic flux within the coupling, increases the available torque transfer by around $40 \%$ for a given magnet set. The complete outer coupling is mounted on ceramic water-lubricated bearings and attached directly to the propeller assembly. The chosen radial arrangement allows for a shorter design (Pugi et al., 2018) than an axial one, which was a major consideration from the point of view of the internal arrangement of the BRIDGES glider. It also allows the gap between the two sets of magnets to be minimised, improving the torque capabilities of the coupling for the same number of magnets used (Allotta et al., 2015).

The thruster unit is designed to operate at a maximum output of $100 \mathrm{~W}$ with supply voltage of up to $60 \mathrm{~V}$ and maximum rpm of 1000 , thanks to the use of a two-stage, 10:1 gearbox. This makes it oversized for the needs of the current vehicle, but should make it capable of coping with the subsequent incarnations of the AUV with inevitably larger amounts of payloads and drag. Given its wide operating range, the thruster could also be fitted to other vehicles, reducing development costs and improving interoperability and maintainability. To further improve the latter functionality, the thruster has been designed to provide an easy attachment and detachment of the hub and blades with basic hand tools, fast replacement of electronics with only a single seal having to be re-done, and straightforward mounting and removal from the vehicle by using external clamps. The integrated electronics are designed to be controlled through the RS-232 serial protocol compatible with many contemporary underwater vehicle platforms, adding to the interoperability of the thruster.

To improve in-service reliability of the unit, it has been designed to provide a high heat rejection rate from the transistors and other key electronic components. The robustness of the thruster was also verified through endurance tests carried out in an environmental chamber at correct pressure and temperature corresponding to the ocean bottom conditions. 


\section{Methodology}

\subsection{Test matrix}

Three main sets of tests have been carried out on the thruster in order to quantify its performance in realistic design conditions:

1. Brake tests - involved applying a known torque to the propeller shaft and measuring the power consumption over a range of rpm; the results were used to estimate the net efficiency of the motorgearbox system as well as to validate the design of the magnetic coupling.

2. Bollard pull tests - the thruster unit was held still and fixed rpm demands were sent to it; thrust developed by each of the tested propellers, total power consumption, and blade tip deflection were measured.

3. Tests at speed-power consumption and developed thrust were measured for each propeller at sprint and cruise speed equivalents for a range of rpm; tare measurements with no propeller were also acquired to correct the data.

For each category of tests, rpm values up to the maximum of 1000 were used, except in cases where the resultant power output would exceed maximum output of the thruster. There the maximum possible rpm was typically in the range between 900 and 1000 .

The presently tested propulsion unit was designed to work in ahead direction only. Moreover, due to the propeller having the passive folding capability, it could not be rotated in reverse with the vehicle moving ahead without folding. Consequently, two forward advance speeds were chosen for the towed tests: 0.13 and $0.44 \mathrm{~m} / \mathrm{s}$. These were aimed to replicate the mean nominal inflow speeds at cruise and sprint speeds of 0.25 and $1.00 \mathrm{~m} / \mathrm{s}$. At each speed, measurements were carried out over a range of rpm, starting from high loading and extending to slightly negative thrust coefficient regime.

Wake fraction relating the two speeds was predicted using numerical simulations discussed by Lidtke et al. (2017). In reality, the advance speed seen by the propeller at the cruise condition is expected to be $0.11 \mathrm{~m} / \mathrm{s}$, but it was not possible to achieve this value during the tests due to the limitations of the towing tank carriage system. Hence, the nearest possible value was opted for. In service, this would translate to a vehicle speed of approximately $0.3 \mathrm{~m} / \mathrm{s}$, or $0.05 \mathrm{~m} / \mathrm{s}$ above target cruise velocity.

\subsection{Experimental apparatus}

The present experiments were carried out at the Southampton Solent University towing tank. This facility is $60 \mathrm{~m}$ long, $3.7 \mathrm{~m}$ wide, and $1.8 \mathrm{~m}$ deep. These dimensions suffice for it to be used for sailing yacht and fast planing craft testing and were more than sufficient to carry out the current propeller tests without significant blockage effects.

The thruster unit was mounted in a hydrodynamic fairing made of acrylic and 3D printed elements. The fairing was then connected to a $40 \times 40 \mathrm{~mm}$ cross-section Rexroth beam. At its upper end, the beam was connected to a two-component dynamometer placed on the towing tank carriage. To minimise its resistance, the vertical beam was also placed in a foam and plywood fairing. In the final configuration, the shaft axis of the propeller was placed $433 \mathrm{~mm}$, or 1.7 propeller diameters, below the water surface. This is greater than used for canonical propeller tests (SVA Hydrodynamic Solutions, 2015) and hence was considered sufficient to minimise free-surface interactions.

Propellers were tested in a forward-looking configuration as reliably emulating the full-scale inflow conditions they would see in the wake of the vehicle was not feasible within the scope of the investigation. It was therefore decided to focus on achieving the correct mean nominal inflow speed and Reynolds number with clean, undisturbed inflow conditions. The neglected effect of thrust deduction, i.e. change of hull



Fig. 5. View of the experimental rig used to test the thruster unit in the towing tank. Thruster is placed in a transparent fairing with a 3D-printed end cap. The white part of the fairing houses the supporting strut and wiring. Flow from left to right, i.e. the thruster is in an open-water configuration.

resistance due to propeller suction, was expected to be low due to the relatively low loading and associated pressure drop induced by the propeller. The final test assembly is depicted in Fig. 5.

The dynamometer used allowed the net axial and side forces acting on the test assembly to be measured. The side force was only used to confirm accurate alignment of the thruster, while the axial force included both the useful thrust of the propeller and the drag of the assembly. Voltage and current supply to the unit were also measured to estimate the consumed power. While it would have been beneficial to also measure the propeller torque directly, this could not be feasibly achieved while also testing the actual thruster unit without substantial modifications that were out of the scope of the current experimental campaign.

Force data was non-dimensionalised using a standard notation, whereby the non-dimensional speed, or advance coefficient, was computed as

$$
J=\frac{V_{A}}{n D},
$$

given the nominal inflow speed, $V_{A}$, revolutions per second, $n$, and propeller diameter, $D$. The propeller thrust coefficient could then be computed using

$$
K_{T}=T\left(\rho n^{2} D^{4}\right)^{-1},
$$

where $\rho$ was computed as a function of the measured tank temperature following the standard ITTC values (ITTC, 2011). This yielded density of $999.024 \mathrm{~kg} / \mathrm{m}^{3}$ and kinematic viscosity of $1.125 \cdot 10^{-6} \mathrm{~m}^{2} / \mathrm{s}$ at 15.5 degrees Centigrade. Although the propeller torque could not be directly measured, the power consumed by the thruster and its rpm were. As the motor-gearbox efficiency was measured during the brake tests, for a known rpm and absorbed power the motor performance curves could be interpolated to back-calculate the torque delivered to the propeller. The resulting value could be non-dimensionalised,

$$
K_{Q}=Q\left(\rho n^{2} D^{5}\right)^{-1},
$$

and open-water efficiency computed,

$$
\eta_{0}=\frac{J K_{T}}{2 \pi K_{Q}} .
$$

It has been found that for lower speeds and rpm values, this procedure was not accurate enough to yield reliable results due to the minimum resolution of the current transducer used. However, at higher revolutions the propeller torque and thruster power consumption were sufficiently high to be measured accurately, yielding repeatable and consistent efficiency figures.

In order to measure the tip deflections of the propellers, an underwater camera was mounted at the same depth as the shaft with the field 


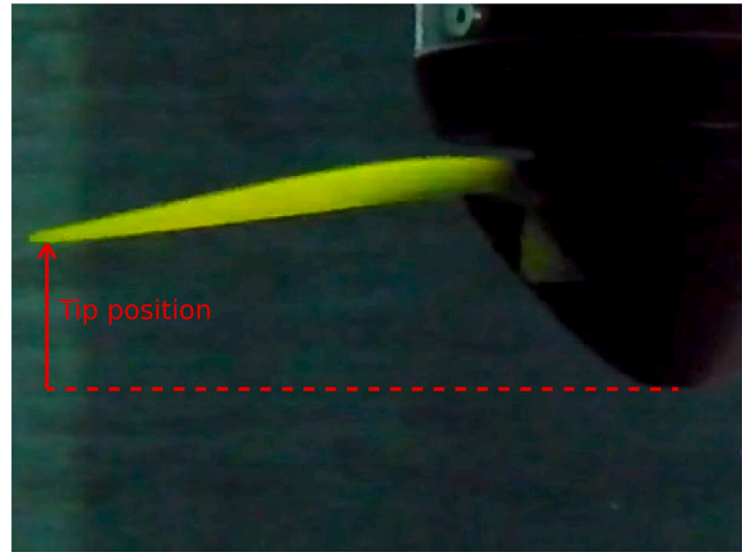

Fig. 6. An example frame used to perform tip deflection measurement. End of the hub aws used as a fixed reference point.

of view overlooking the propeller plane. Its imagery was calibrated by placing a know-size target where the propeller blades would be and computing a transfer function between the image pixels and distance. By knowing the initial, unloaded position of the blades, tip deflection could be computed as shown in Fig. 6. With a more sophisticated set-up it could be possible to carry out more precise measurements, including full-field deformation and blade twist (Banks et al., 2015). However, the present apparatus could only be reliably used to measure average deflections of the tip of the propeller as its estimated resolution was of the order of $0.2 \mathrm{~mm}$, resulting in approximately 3-5 pixels at the finest parts of the blade close to the tip, which was insufficient to deduce more information. Furthermore, the set-up could only be used reliably in the bollard-pull condition.

\subsection{Data acquisition and processing}

In order to measure the forces, deflection of the dynamometer was acquired using LVDT transducers which translate a linear displacement into a voltage signal. That was recorded at a rate of $100 \mathrm{~Hz}$; in order to obtain average signals, data was windowed based on the moving average and standard deviation in order to select steady acquisition periods. For most runs these lasted approximately $30 \mathrm{~s}$, with longer runs recorded deliberately for towed tests at the lower investigated speed. This helped to improve signal-to-noise ratio and led to more reliable results. Only average power consumption of the thruster was recorded for each run using precision voltage and current meters.

In order to differentiate between the thrust of the propeller and drag of the test fairing, tare drag measurements were carried out and their results were subtracted from the total acquired force. To ensure the validity of the correction applied in this way, each measurement was repeated three times to minimise the likelihood of non-systematic errors.

Tip deflection were computed using the open-source Kinovea software by tracking the position of the tip as it passed the measurement region. For each rpm, images acquired at 20 fps over a period of $10 \mathrm{~s}$ were processed and an average figure was computed.

\subsection{Self-propulsion estimation}

The measured open-water propeller characteristics were compared against resistance data of the BRIDGES glider AUV, reported in Lidtke et al. (2018), in order to determine the self-propelled power and rpm. This was done by assuming a given speed of the vehicle and computing thrust of the propeller for different rpm at that condition, having accounted for the wake fraction, $w_{t}$, modifying the inflow to the propeller. This was determined using Pitot tube measurements, carried out alongside the force measurements, and numerical simulations from Lidtke et al. (2017). An intersection point was then found at which thrust was equal to the resistance of the vehicle. Due to the fact that simulations with a propeller model were not carried out, the effect of the action of the propeller on the resistance was neglected, thus assuming thrust deduction of $t=0$. A safety margin of $50 \%$ was also applied to the thrust to account for possible uncertainty in the predicted resistance and propeller performance. This was determined based on typical AUV service data (Rudnick et al., 2004; Eriksen et al., 2001; Graver, 2005; Phillips et al., 2010). Thus, the total propulsive efficiency could be estimated as:

$$
\eta_{T}=\eta_{0} \eta_{H} \eta_{R} \eta_{M},
$$

where $\eta_{H}=\frac{1-t}{1-w_{t}}$ is the hull efficiency, $\eta_{R}$ is the relative rotative efficiency assumed to be 1 due to low loading and small blade area ratio of the current propeller, and $\eta_{M}$ is the motor-gearbox efficiency.

\section{Results}

\subsection{Motor-gearbox performance}

Fig. 7 presents the results obtained from the brake tests on the thruster. It depicts the power delivered by the motor subject to constant torque across a range of rpm, as well as its efficiency computed as a ratio of the useful power, $P_{O U T}$, and absorbed electrical power, $P_{e}$,

$$
\eta_{M}=\frac{P_{O U T}}{P_{e}}=\frac{2 \pi Q n}{U_{S U P} I_{S U P}} .
$$

In the above, $U_{S U P}$ is the supply voltage, and $I_{S U P}$ is the current drawn from the power source. Tests were carried out at supply voltage level of $54 \mathrm{~V}$.

Measured data show that the variation in power follows the expected linear trend, as judged from the relative fit quality of the linear regression curves. This indicates that if the consumed electrical power and rpm may be accurately measured during the propeller tests, the obtained motor performance can be used to calculate the torque developed by the propeller. The results also show that the efficiency of the motor-gearbox combination plummets as the applied torques become low, mainly due to mechanical losses in the two-stage gearbox overcoming the useful load. Furthermore, at higher rpm the efficiency of the motor increases for the same torque and the performance curve is much flatter than when operating at lower revolutions per second, highlighting the advantage of running the thruster close to its design power and rpm.

\subsection{Bollard pull}

Results of the bollard pull tests are depicted in Fig. 8. Based on the basic propeller theory, described by Eqs. (2) and (3), the thrust and power curves were expected to follow a quadratic and cubic trend with the shaft rate, respectively. However, in both cases, a deviation from this trend is observed at high revolutions, as visible from comparing the measured data to linear trend lines fitted to the values obtained at lower $\mathrm{rpm}$. This deviation from the expected trend appears more pronounced for the propellers characterised by lower $\mathrm{P} / \mathrm{D}$, which produce less thrust but can reach higher rpm without the thruster reaching its maximum power. It was suspected that the root cause of this was deformation of the propeller blades as loading is increased, which prompted the deflection measurements presented in Section 4.4. 




(a) Useful output power, $P_{\text {OUT }}=2 \pi n Q$

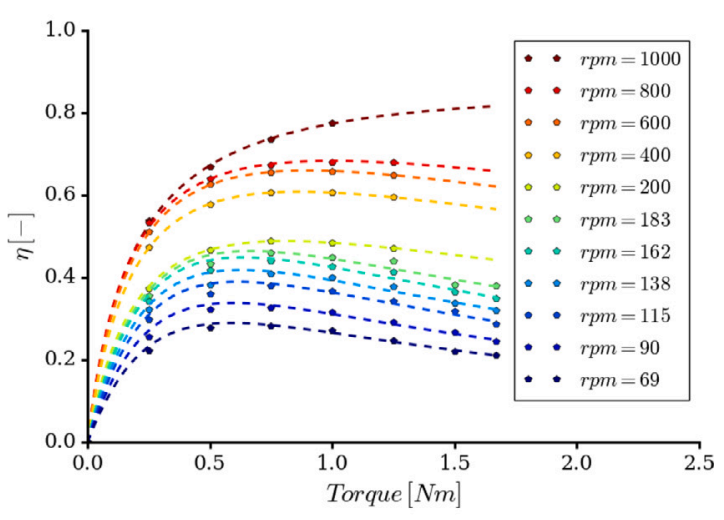

(b) Efficiency, defined by Eq. (6)

Fig. 7. Output power and computed efficiency of the motor-gearbox combination measured during the brake tests. Markers indicate data points and dashed lines are linear and polynomial fits to data.

\subsection{Propeller performance at speed}

From the point of view of allowing an AUV achieve self-propulsion at the correct design speed, the most critical information is the thrust developed by the propeller; this information is presented in Fig. 9 for speeds corresponding to the cruise and sprint conditions. Data reveal an overall satisfactory repeatability obtained during repeat tests carried out over several measurement series. One observation to the contrary are outliers seen at the lowest recorded J-values for the tests corresponding to the sprint condition; during those measurement runs the thruster reached its maximum power and likely could not achieve the desired rpm, leading to a drop in thrust. These points were therefore ignored when fitting the trend lines.

Results indicate that as the advance speed increases, the advance coefficient at which zero thrust is produced also goes up, increasing the effective range of operation of the propeller. Increasing the speed also causes the thrust curves to deviate from a linear trend. However, for a fixed speed, propeller theory would suggest a linear change of $K_{T}$ with $J$, as seen in Eqs. (1) and (2). A rapid change in variation of thrust was also measured for the propeller with the highest pitch at lower speed.

Changing the advance speed from 0.44 to $0.13 \mathrm{~m} / \mathrm{s}$ leads to a proportional reduction in propeller rpm for equivalent $J$-values. Since the velocity seen by a blade section at non-dimensional radial ordinate $x$ may be written as

$$
V_{\text {ref }}=\sqrt{V_{a}^{2}+(2 \pi x R n)^{2}}
$$



(a) Thrust

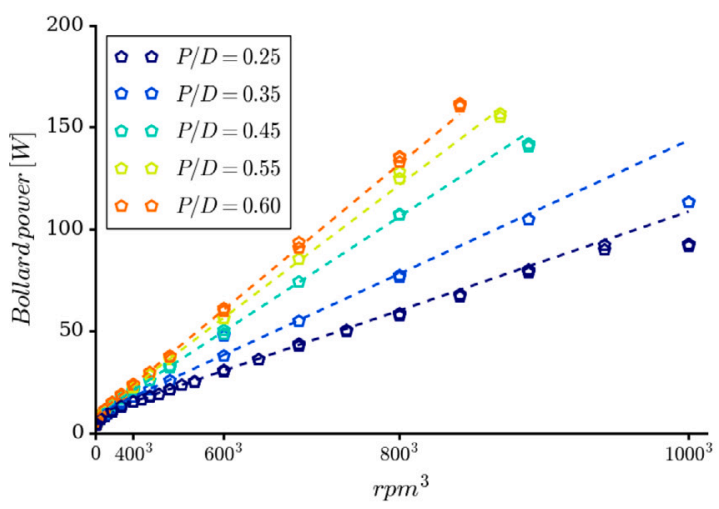

(b) Absorbed power, $P_{e}$

Fig. 8. Thrust and power absorbed by the thruster in bollard pull conditions. Markers indicate measurement points and dashed lines are linear trend lines fitted to data below $600 \mathrm{rpm}$.

if propeller-induced velocities are neglected to a first approximation, it is expected that the local Reynolds number experienced by it will also change. This is explored in Fig. 11 which depicts local Reynolds number for the tested propellers at $\mathrm{x} / \mathrm{R}$ of 0.7 , or the location of nominal blade pitch. This shows that between the two tested conditions Re takes values of the order of 50,000 and 100,000, which corresponds to typical values at which laminar-turbulent transition is observed (Selig et al., 1995). This range of Reynolds numbers has also been reported to cause significant performance variations in model-scale ship propellers (Heinke et al., 2019; Li et al., 2019).

In order to better visualise the effect of changing Reynolds number on the propellers, thrust coefficient values of all for the investigated conditions are plotted simultaneously in Fig. 10. Doing so shows that for the same propeller drastic changes are observed in the measured forward thrust between the two speed regimes. J-range for which positive thrust has been measured is extended by approximately $30 \%$ for all of the P/D values when Re is increased. At lower speeds, however, the produced thrust is lower at the higher speed of advance. Thrust curves also show a steeper gradient as the propellers approach near-bollard-pull conditions.

Due to very low torques developed by the propeller at the slower advance speed, seen in Fig. 13, the resultant power consumption of the thruster did not differ significantly from the tare values measured with the hub spinning without the blades. Consequently, efficiency of the propeller could not be accurately measured in this condition aside from data points close to the bollard pull condition for which relatively high torques were developed by the propellers. At the higher speed, however, relatively repeatable results were obtained, as seen in Fig. 14. 




(a) $\mathrm{V}_{A}=0.13 \mathrm{~m} / \mathrm{s}$ (cruise condition)

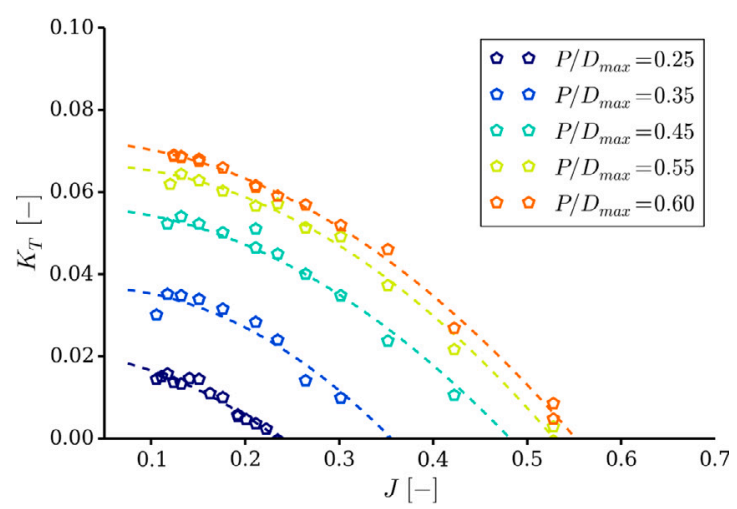

(b) $\mathrm{V}_{A}=0.44 \mathrm{~m} / \mathrm{s}$ (sprint condition)

Fig. 9. Thrust coefficient measured for the tested propellers at two different advance speeds and across a range advance coefficients. Dashed lines are quadratic polynomials.



Fig. 10. Effect of Reynolds number on the thrust coefficient developed by the propellers.

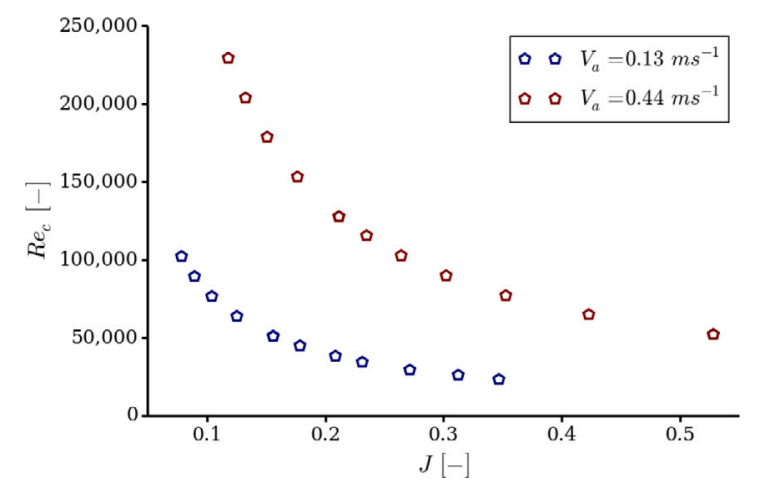

Fig. 11. Local Reynolds number at non-dimensional radius of 0.7 for propeller with $\mathrm{P} / \mathrm{D}$ of 0.45 .

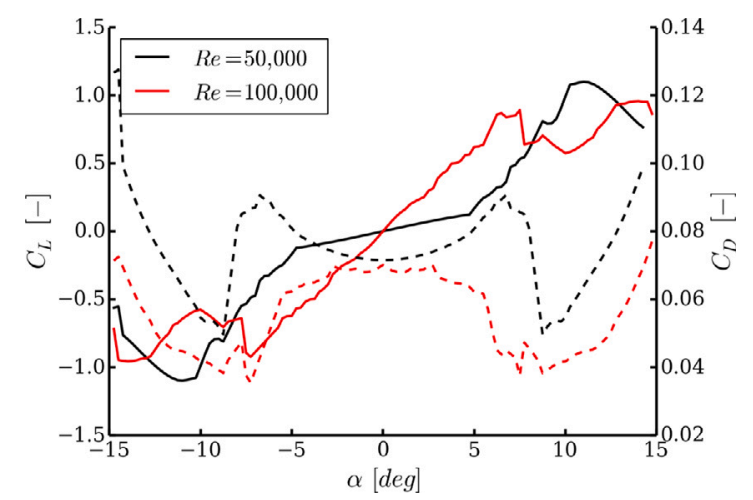

Fig. 12. Lift (solid lines) and drag (dashed lines) coefficients of the blade section at two typical Reynolds numbers computed with Xfoil.

The only deficiency in the latter data are the outliers appearing after the propeller had stalled; these are, however, relatively unimportant from the point of view of vehicle propulsion.

In order to allow self-propelled analysis at the lower advanced speeds, regressed torque coefficient data obtained at the sprint condition was scaled by assuming most of the changes to $K_{Q}$ would be related to the drag variation of the chosen blade section with Reynolds number. For the highest pitched propeller, the relative change estimated based on the data obtained at low J-values indicated a relative increase of $K_{Q}$ of $32 \%$ and $55 \%$ for the lowest pitch, with an approximately linear variation over tested $\mathrm{P} / \mathrm{D}$ values. This is qualitatively consistent with Xfoil (see: Drela, 1989) 2D section data estimates seen in Fig. 12, which indicate a change in $C_{D}$ between 5 and $20 \%$, depending on the angle of attack. Discrepancy between the measured and expected values may be explained by inaccuracies in capturing transition effects using Xfoil and presence of three-dimensional effects.

Despite the need to apply the afore-mentioned assumptions, the collected data indicate that, as expected from propeller theory, increasing the $\mathrm{P} / \mathrm{D}$ ratio leads to an increased efficiency and a wider range of viable J-values. However, beyond P/D 0.45, the observed increases are relatively small. The maximum efficiency values predicted at the lower advance speed are also considerably lower.

\subsection{Blade tip deflection in bollard pull}

Fig. 15 presents propeller tip deflections measured in bollard-pull conditions. Data show that at low revolutions the amount of blade bending is relatively small compared to the propeller diameter, reaching only $2 \%$ at rpm of 400 . However, beyond that point all of the tested blades show a sharp increase in the amount of bending, reaching between 6 and $10 \%$ of diameter at highest rpm. A trend is also seen 


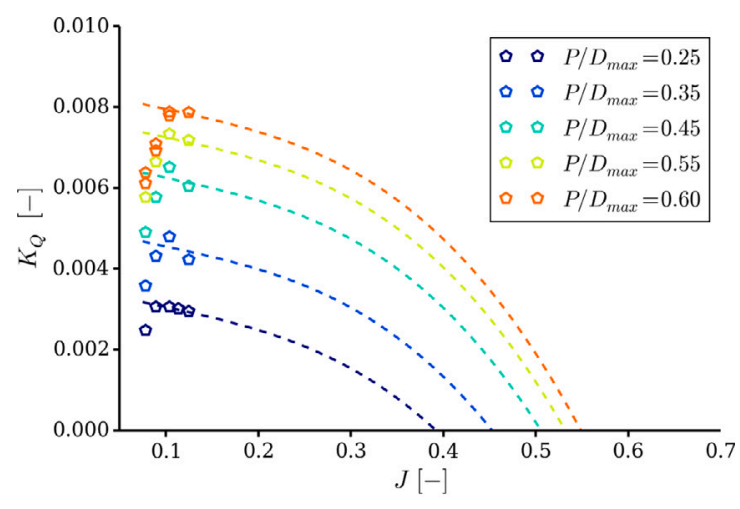

(a) $\mathrm{V}_{A}=0.13 \mathrm{~m} / \mathrm{s}$ (cruise condition)

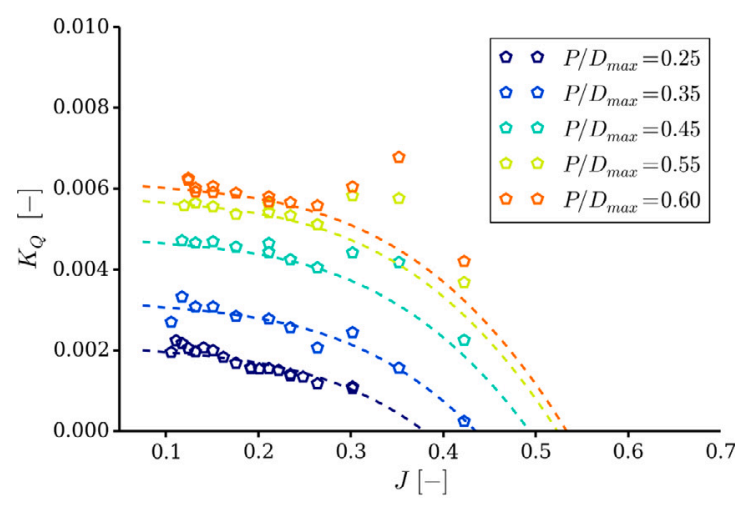

(b) $\mathrm{V}_{A}=0.44 \mathrm{~m} / \mathrm{s}$ (sprint condition)

Fig. 13. Propeller torque coefficient measured for the tested propellers at two different advance speeds and across a range advance coefficients. Dashed lines are cubic polynomials.

whereby the higher the pitch of the propeller, the more it deflects. A relevant observation is also that albeit the measurement method used is relatively simple, the variability of data is smaller than the trends observed in the results.

\subsection{Predicted self-propelled performance}

A final set of data is depicted in Figs. 16 and 17, which present selfpropelled operating points for the BRIDGES AUV predicted using the measured propeller performance curves and resistance data discussed in Lidtke et al. (2018). At the sprint condition, the motor-gearbox efficiency term is seen to be by up to $12 \%$ higher for the lowerpitched propellers due to the self-propelled rpm being higher. This effect is offset by the propellers with higher P/D exhibiting higher hydrodynamic efficiencies, although this only occurs for pitch-diameter ratios up to 0.45 . At the cruise condition, motor efficiency is by far the lowest component of total efficiency, ranging between 10 and $20 \%$, and decreases with P/D because of the associated decrease in rpm at self-propulsion. Nevertheless, the increase in propeller open-water efficiency associated with higher pitch is also seen at this speed and offsets the small relative drop motor efficiency, leading to the propellers with higher $\mathrm{P} / \mathrm{D}$ requiring less power at self-propulsion. However, due to the dominant effect of the motor-gearbox efficiency term at the lower speed, the maximum net efficiency of the propulsive subsystem has been predicted to be only $7 \%$, compared to $38 \%$ at the design condition. Figures of total delivered electrical power also reveal that at the cruise condition a distinct optimum exists at $\mathrm{P} / \mathrm{D}$ of 0.45 . At the higher speed this is not visible, but that might be due to the relatively coarse offsets of the independent variable.



(a) $\mathrm{V}_{A} 0.13 \mathrm{~m} / \mathrm{s}$ (cruise condition)

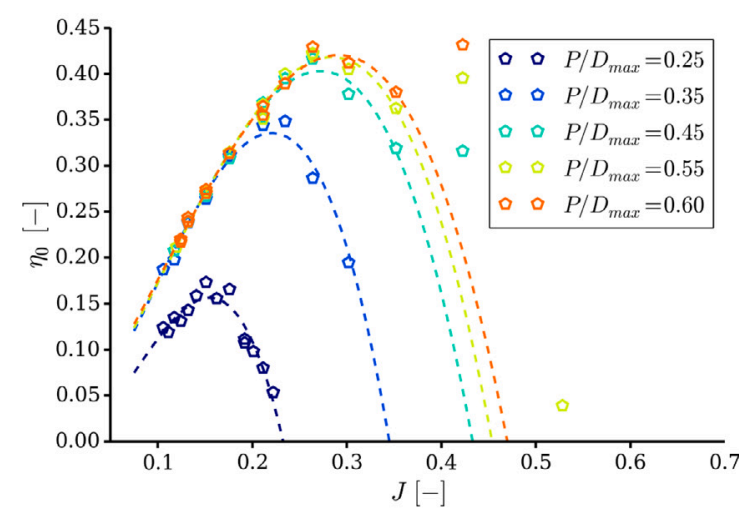

(b) $\mathrm{V}_{A} 0.44 \mathrm{~m} / \mathrm{s}$ (sprint condition)

Fig. 14. Open water efficiency measured for the tested propellers at two different advance speeds and across a range advance coefficients. Dashed lines are cubic polynomials.

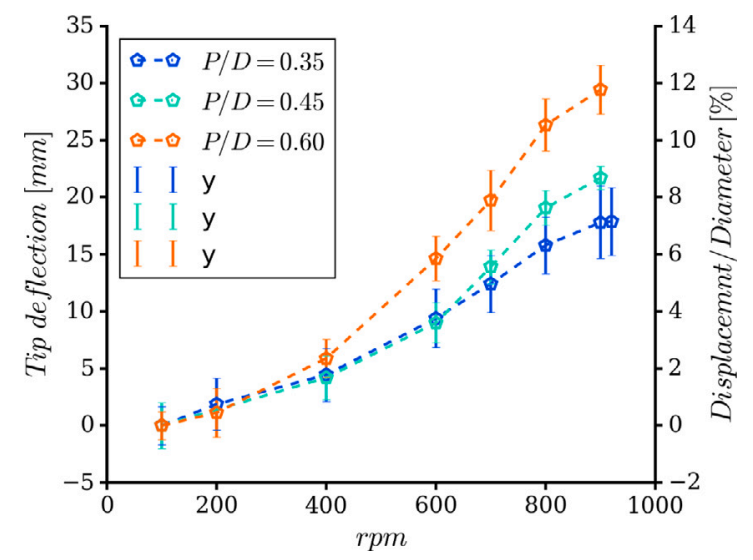

Fig. 15. Tip deflections measured in bollard pull conditions. Error bars indicate a confidence interval of one standard deviation on either side of the mean value indicated with the markers.

\section{Discussion}

Hydrodynamic efficiency of the tested propellers has been shown to vary significantly across the Reynolds number regime spanning the expected operational profile of the BRIDGES vehicle. At lower advance speeds the blades were measured to produce more torque and less thrust, which is consistent with the expectations derived from 2D foil section performance. Furthermore, at lower advance speeds the propellers were observed to undergo stall at advance coefficients 


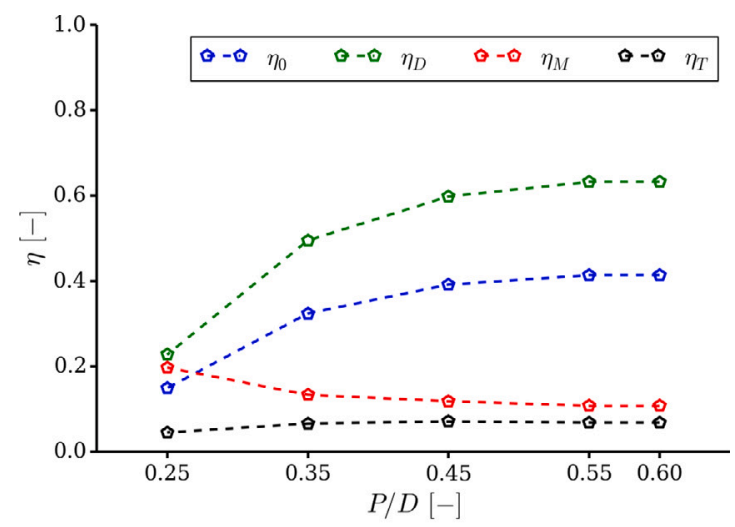

(a) $V_{S}=0.3 \mathrm{~m} / \mathrm{s}$

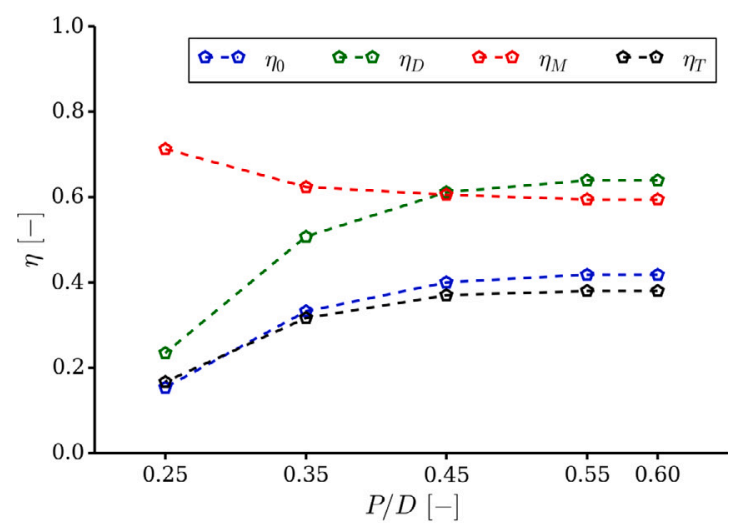

(b) $V_{S}=1.0 \mathrm{~m} / \mathrm{s}$

Fig. 16. Self-propelled efficiencies predicted for the BRIDGES Deep Explorer AUV moving at sprint and cruise forward speeds.

approximately 15 to $20 \%$ lower than at the investigated sprint condition. This may be attributed to the presence of a "dead zone" in the lift coefficient curve around small angles of attack at low Reynolds numbers. These trends are also consistent with data reported by Brandt and Selig (2011) for aerial vehicle propellers tested in air at similar Reynolds numbers.

The tested propellers were designed using blade element momentum theory (BEMT), which is built upon integration of $2 \mathrm{D}$ section performance along the blade and thus should account for low-Re behaviour. However, the experimental results indicated that the propellers with lower P/D suffered more loss of thrust at lower speeds than the initial analysis had indicated, leading to worse overall efficiency.

The net propulsive efficiency has also been significantly affected by the variability of the combined motor-gearbox efficiency with rpm and applied torque. This has been shown to dominate the self propulsion efficiency at lower speeds, favouring higher propeller revolutions above a minimum torque threshold. In general, this contradicts with the hydrodynamic requirements which push the propeller towards lower revolutions and higher pitch, but this is also further affected by the non-linear variations induced by the Reynolds number.

Blade deflection has also been shown to reduce thrust developed by the propellers as the advance coefficient is reduced. This is tied to the loading on the blade and therefore becomes more significant at higher revolutions and advance speed when the net force developed by the propeller becomes higher. Similar trends were reported in the work by Pawar and Brizzolara (2019) who studied performance of a 3D printed conventional propeller. In the case of the current propellers, a critical rpm value of 500 has been identified in both thrust data and measured tip deflections. As close to the optimum efficiency point

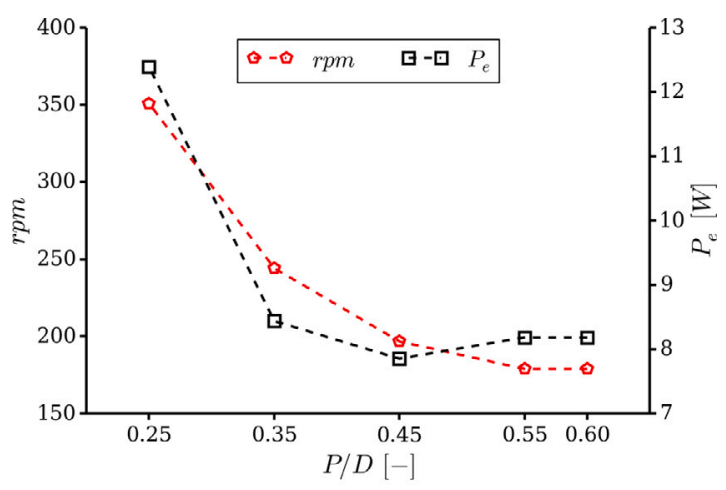

(a) $V_{S}=0.3 \mathrm{~m} / \mathrm{s}$

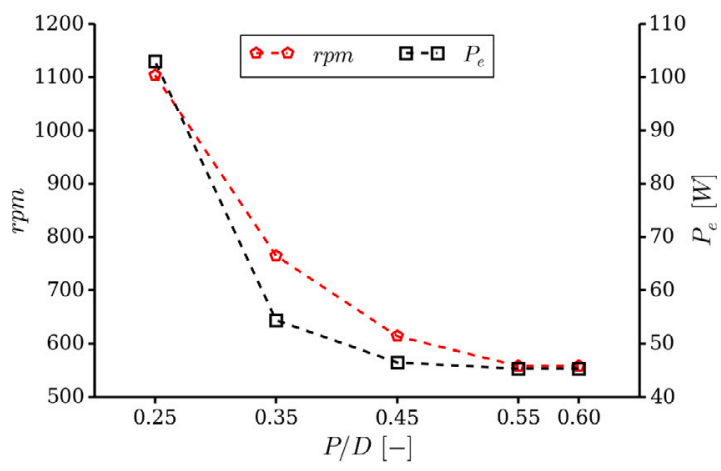

(b) $V_{S}=1.0 \mathrm{~m} / \mathrm{s}$

Fig. 17. Self-propelled power and rpm predicted for the BRIDGES Deep Explorer AUV vehicle moving at sprint and cruise forward speeds.

propellers operate at relatively low load factors, this phenomenon does not appear to affect the self-propelled operating point significantly, but might play a role in off-design conditions or on vehicles such as hover-type AUV's or ROV, whose thrusters operate in near bollard-pull conditions most of the time.

\section{Conclusions}

Presented results have shown that the final performance of the investigated thruster unit fitted with propellers of various pitch-diameter ratios varies significantly with changing Reynolds number, thrustinduced blade deformation, and motor-gearbox efficiency dependent on the chosen operating point. It has been shown that the electromechanical efficiency may drop as low as $10 \%$ at slow advance speeds and at correspondingly low propeller revolutions and torque levels. This loss of performance at low speed is further exacerbated by the decrease in hydrodynamic efficiency induced by the low Reynolds number effects, such as increased section drag coefficient and lower lift curve slope at low angles of attack. Thus, all of the aforementioned factors have severe implications on the interplay of hydrodynamic and electro-mechanical efficiencies governing the net propulsive efficiency. Nevertheless, results have confirmed that despite a very wide operational profile required of the designed propulsor, the same fixed-pitch propeller may be used to actuate the vehicle in the design configuration while using less than 10 and $100 \mathrm{~W}_{e}$ at cruise and sprint conditions, respectively. The ultimate validation of the thruster was achieved during the field trials of the BRIDGES glider, during which both the speed and power consumption requirements have been met. Moreover, an upscaled version of the unit with a $600 \mathrm{~W}$ power rating is now also being deployed on the new A2KUI under-ice AUV.

The experiments have also indicated that the adopted selective laser sintering (SLS) 3D printing method allows for robust propeller 
blades to be rapidly manufactured. This could be leveraged to quickly replace broken components at sea and even to adjust the propeller pitch depending on particular vehicle configuration if, for instance, much higher drag is expected due to the fitting of additional external payloads. Furthermore, the measurements have indicated that hydroelasticity plays a significant role in governing performance of propellers manufactured using the selected method. In the present study, this phenomenon was treated as a constraint to be overcome. However, future applications could investigate harnessing blade deformation to better control performance of the propeller across different operating regimes, for instance, by exploiting bend-twist coupling to passively adjust the pitch of the propeller to the current thruster set point, consequently improving overall propulsive efficiency of the entire vehicle.

\section{CRediT authorship contribution statement}

Artur K. Lidtke: Design of experimental rig, Measurements, Data analysis, Writing - review \& editing. Nicholas P. Linton: Design of thruster, Measurements, Writing - review \& editing. Hannah L. Wright: Design of thruster, Experimental apparatus. Stephen R. Turnock: Supervision, Design of experimental rig, Writing - review \& editing. Jon Downes: Supervision, Writing - review \& editing.

\section{Declaration of competing interest}

The authors declare that they have no known competing financial interests or personal relationships that could have appeared to influence the work reported in this paper.

\section{Acknowledgements}

The presented work has been funded as a part of the BRIDGES Project (http://www.bridges-h2020.eu/) under the European Union's Horizon 2020 research and innovation grant agreement No 635359. We also wish to thank the colleagues who provided help during the towing tank test campaign.

\section{References}

Allotta, B., Costanzi, R., Gelli, J., Pugi, L., Ridolfi, A., 2015. Design of a modular propulsion system for marta auv. In: OCEANS 2015 - Genova. pp. 1-7. http: //dx.doi.org/10.1109/OCEANS-Genova.2015.7271397.

Allotta, B., Pugi, L., Bartolini, F., Ridolfi, A., Costanzi, R., Monni, N., Gelli, J., 2015. Preliminary design and fast prototyping of an autonomous underwater vehicle propulsion system. Proc. Inst. Mech. Eng. M: J. Eng. Marit. Environ. 229 (3), 248-272. http://dx.doi.org/10.1177/1475090213514040.

Banks, J., Giovannetti, L.M., Soubeyran, X., Wright, A., Turnock, S., Boyd, S., 2015. Assessment of digital image correlation as a method of obtaining deformations of a structure under fluid load. J. Fluids Struct. 58, 173-187. http://dx.doi.org/10. 1016/j.jfluidstructs.2015.08.007.

Brandt, J.B., Selig, M.S., 2011. Propeller performance data at low reynolds numbers. In: 49th AIAA Aerospace Sciences Meeting, Orlando, Florida, USA, 4-7 Jan.

Carlton, J.S., 2012. Marine Propellers and Propulsion. Butterworth-Heinemann, Oxford.

Chen, Z., Yu, J., Zhang, A., Zhang, F., 2016. Design and analysis of folding propulsion mechanism for hybrid-driven underwater gliders. Ocean Eng. 119 (May), 125-134. http://dx.doi.org/10.1016/j.oceaneng.2016.03.034.

Claus, B., Bachmayer, R., Williams, C.D., 2010. Development of an auxiliary propulsion module for an autonomous underwater glider. Part M: J. Eng. Marit. Environ. 224, 255-266. http://dx.doi.org/10.1243/14750902JEME204.
Drela, M., 1989. Xfoil: An analysis and design system for low reynolds number airfoils. In: Mueller, T.J. (Ed.), Low Reynolds Number Aerodynamics. Springer Berlin Heidelberg, Berlin, Heidelberg, pp. 1-12.

Eriksen, C.C., 2003. Autonomous Underwater Gliders. In: Autonomous and Lagrangian Platforms and Sensors (ALPS) Workshop, Sea Lodge, La Jolla, CA, USA, 31 March - 2 April, pp. 1-5.

Eriksen, C.C., Osse, T.J., Light, R.D., Wen, T., Lehman, T.W., Sabin, P.L., Ballard, J.W., Chiodi, A.M., 2001. Seaglider : A long-range autonomous underwater vehicle for oceanographic research. IEEE J. Ocean. Eng. 26 (4), 424-436.

Giovannetti, L.M., Banks, J.F., Boyd, S.W., Turnock, S.R., 2016. Developing tools for assessing the fluid structure interaction of passive adaptive composite foils. In: Sixth International Conference on Structural Engineering, Mechanics and Computation, South Africa. 05 - 07 Sep.

Giovannetti, L.M., Banks, J., Ledri, M., Turnock, S., Boyd, S., 2018. Toward the development of a hydrofoil tailored to passively reduce its lift response to fluid load. Ocean Eng. 167, 1-10.

Graver, J.G., 2005. Underwater gliders - dynamics, control and design (Ph.D. thesis). Princeton University.

Heinke, H.-J., Hellwig-Rieck, K., Lübcke, L., 2019. Influence of the reynolds number on the open water characteristics of propellers with short chord lengths. In: Sixth International Symposium on Marine Propulsors, SMP'19, Rome, Italy.

Isa, K., Arshad, M., Ishak, S., 2014. A hybrid-driven underwater glider model, hydrodynamics estimation, and an analysis of the motion control. Ocean Eng. 81, 111-129. http://dx.doi.org/10.1016/j.oceaneng.2014.02.002.

ITTC, 2011. ITTC - Recommended Procedures and Guidelines: Fresh Water and Seawater Properties. Technical Report.

Kim, J., Chung, W.K., 2006. Accurate and practical thruster modeling for underwater vehicles. Ocean Eng. 33 (5), 566-586. http://dx.doi.org/10.1016/ j.oceaneng.2005.07.008, URL: https://www.sciencedirect.com/science/article/pii/ S0029801805001836.

Li, D.-Q., Lindell, P., Werner, S., 2019. Transitional flow on model scale propellers and their likely influence on performance prediction. In: Sixth International Symposium on Marine Propulsors, SMP'19, Rome, Italy.

Lidtke, A.K., Turnock, S.R., Downes, J., 2017. Hydrodynamic design of underwater gliders using k-kl-omega rans transition model. IEEE J. Ocean. Eng. August, 1-13. http://dx.doi.org/10.1109/JOE.2017.2733778.

Lidtke, A.K., Turnock, S.R., Downes, J., 2018. Characterising influence of transition to turbulence on the propulsive performance of underwater gliders. In: 32nd Symposium on Naval Hydrodynamics (SNH), Hamburg, Germany, 5-10 Aug.

Maljaars, P., Bronswijk, L., Windt, J., Grasso, N., Kaminski, M., 2018. Experimental validation of fluid-structure interaction computations of flexible composite propellers in open water conditions using bem-fem and rans-fem methods. J. Mar. Sci. Eng. 6 (2), 51.

Molland, A., Turnock, S.R., Hudson, D., 2011. Ship Resistance and Propulsion: Practical Estimation of Propulsive Power. Cambridge University Press.

Pawar, S., Brizzolara, S., 2019. Hydroelastic analysis of 3d printed marine propeller working at low reynolds numbers. In: Sixth International Symposium on Marine Propulsors, SMP'19, Rome, Italy.

Phillips, A.B., Turnock, S.R., Furlong, M., 2010. The use of computational fluid dynamics to aid cost-effective hydrodynamic design of autonomous underwater vehicles. Proc. IMechE Part M: J. Eng. Marit. Environ. 224, 239-254. http://dx. doi.org/10.1243/14750902JEME199.

Pugi, L., Allotta, B., Pagliai, M., 2018. Redundant and reconfigurable propulsion systems to improve motion capability of underwater vehicles. Ocean Eng. 148, 376-385. http://dx.doi.org/10.1016/j.oceaneng.2017.11.039, URL: https://www. sciencedirect.com/science/article/pii/S0029801817307084.

Rudnick, D.L., Davis, R.E., Eriksen, C.C., Fratantoni, D.M., Perry, M.J., 2004. Underwater gliders for ocean research. Mar. Technol. Soc. J. 38 (2), 73-84. http; //dx.doi.org/10.4031/002533204787522703.

Selig, M.S., Guglielmo, J.J., Broeren, A.P., Giguere, P., 1995. Summary of Low-Speed Airfoil Data - Vol. 1. SoarTech Publications, Illinois, USA.

SVA Hydrodynamic Solutions, 2015. Potsdam propeller test case (PPTC). URL: http: //www.sva-potsdam.de/pptc_data_15.html. 